\title{
The Effects of Biogenic Phosphates on Proteinase-Induced Protein Cleavage and Functioning of Plasminogen Activator
}

\author{
N. S. Pyzhova ${ }^{a}$ and V. N. Nikandrov ${ }^{b, 1}$ \\ ${ }^{a}$ Research Institute of Epidemiology and Microbiology, Ministry of Health of Belarus, Minsk, Belarus \\ ${ }^{b}$ Institute of Physiology, National Academy of Sciences of Belarus, ul. Akademicheskaya 28, Minsk, 220072 Belarus \\ Received July 10, 2007; in final form, July 19, 2007
}

\begin{abstract}
We showed, using the method of lysis of fibrin plates and five substrate proteins in a thin layer of agar gel, that inorganic orthophosphate $(0.001-0.06 \mathrm{M})$ enhances by $50-250 \%$ the activatory functions of streptokinase, urokinase, and tissue plasminogen activator and, in general, by 1.2-12.0 times enhances protein lysis by trypsin, $\alpha$-chymotrypsin, subtilisin, papain, bacterial metalloprotease, and even pepsin at a concentration $<4 \mathrm{mM}$. At higher concentrations, phosphate sharply inhibited pepsin activity and inhibited by $40-50 \%$ gelatin lysis by papain and gelatin (at a peak concentration) and casein lysis by metalloprotease. Inorganic pyrophosphate ions at concentrations of $10^{-8}-10^{-1} \mathrm{M}$ enhanced the cleavage of a number of proteins by serine proteinases and, at concentrations of $10^{-5}-10^{-3} \mathrm{M}$, the activities of pepsin, plasminogen tissue activator, and streptokinase by 100 and $40 \%$, respectively. The pyrophosphate concentrations of $>10^{-3}$ and $>10^{-4} \mathrm{M}$ inhibited pepsinand metalloproteinase-catalyzed lysis of virtually all proteins. ATP increased casein lysis by serine proteinases, metalloproteinase, and pepsin by $20-60 \%$ at concentration of $>10^{-3} \mathrm{M}$ and by $30-260 \%$ at $10^{-2} \mathrm{M}$ concentration. At concentrations of $10^{-2} \mathrm{M}$, it inhibited the cleavage of some proteins by trypsin, chymotrypsin, papain, and metalloproteinase by $20-100 \%$, and, at concentrations of $10^{-3} \mathrm{M}$, lysis of albumin by pepsin and other proteins (except for fibrinogen) by metalloproteinase. A GTP concentration of $10^{-7}-10^{-2} \mathrm{M}$ increased protein degradation by serine proteinases, papain, and gelatin lysis by pepsin by $20-90 \%$, whereas albumin lysis was inhibited by $40-70 \%$. The presence of $10^{-6}-10^{-5} \mathrm{M}$ GTP led to a slightly increased degradation of hemoglobin and casein by bacterial metalloproteinase, while $\geq 10^{-3} \mathrm{M}$ GTP induced a drop in the activity of the metalloproteinase by $20-50 \%$. ADP enhanced gelatin lysis by trypsin, casein lysis by pepsin and papain, and inhibited metalloproteinase activity by $20-100 \%$ (at $\geq 10^{-3} \mathrm{M}$ ). Peculiarities of the effects of AMP and GD(M)P on gelatin lysis were found.
\end{abstract}

Key words: activatory function of streptokinase, urokinase, and tissue plasminogen activator; nucleoside phosphates; orthophosphate; protease activity; pyrophosphate

DOI: $10.1134 / \mathrm{S} 1068162008030163$

\section{INTRODUCTION}

Physicochemical mechanisms of proteolytic reactions and their regulation on molecular and cellular levels are still obscure. ${ }^{2}$ Particularly, the role of inorganic phosphate ions for proteolysis regulation is unclear; despite the apparent triviality of the problem, the published data are fragmentary. It has earlier been shown that mitochondrial fractions of mouse brain and liver did not degrade fibrin. Fibrinolysis only occurred in the presence of $P_{\mathrm{i}}$ [1-3]. Addition of ATP also induced fibrinolytic activity, and in the presence of ADP $+P_{\mathrm{i}}$ the effect of incomplete additivity was observed. It could indicate the stimulation of proteolysis due to ATP resynthesis. However, fibrinolytic activity was also

\footnotetext{
${ }^{1}$ Corresponding author; phone: +375 (17) 284-1759; e-mail: nikandrov@fizio.bas-net.by

${ }^{2}$ Abbreviations: BSA, bovine serum albumin; $P_{\mathrm{i}}$, inorganic orthophosphate; $P P_{\mathrm{i}}$, inorganic pyrophosphate.
}

observed in the presence of a uncoupling agent, 2,4-dinitrophenol, and it was not abolished by cyanide $[2,3]$. Individual lymphoblast cell lines were also demonstrated to increase the proteolytic activity in the presence of $P_{\mathrm{i}}[4]$. According to the data of inhibitory analysis, this effect is not related always to ATP resynthesis. All these facts suggest that there is another way of proteolysis stimulation with $P_{\mathrm{i}}$ independent of ATP resynthesis, and it is the so called "phosphate effect." The nature and occurrence of the phosphate effect remain practically unknown.

In 1987, a specific inhibition of plasminogen-activating function of streptokinase by ATP and 3',5'-AMP was reported: the process was inhibited by $50 \%$ at nucleotide concentrations of $0.1 \mathrm{M}$ [5]. Other nucleotides (ADP, 2'-, 3'-, or 5'-AMP, GTP, UTP, and CTP) did not cause this effect. The attempts to explain this property by the ability of nucleotides to scavenge a 
Table 1. Degradation of substrate proteins with various proteases in a fine layer of agar gel ( $\mathrm{mm}^{2}$ of lysis area)

\begin{tabular}{l|l|l|l|c|c|c}
\hline \multicolumn{1}{c|}{ Substrate } & Trypsin & Chymotrypsin & Subtilisin & Papain & Pepsin & $\begin{array}{c}\text { B. megaterium } \\
\text { metalloprotease }\end{array}$ \\
\hline Fibrinogen & $156 \pm 10$ & $122 \pm 7$ & $274 \pm 18$ & $76 \pm 6$ & $122 \pm 8$ & $118 \pm 7$ \\
Casein & $347 \pm 19$ & $415 \pm 24$ & $512 \pm 33$ & $289 \pm 18$ & $193 \pm 12$ & $388 \pm 14$ \\
Hemoglobin & $140 \pm 5$ & $161 \pm 9$ & $198 \pm 8$ & $83 \pm 11$ & $151 \pm 7$ & $172 \pm 10$ \\
Gelatin & $462 \pm 9$ & $309 \pm 13$ & $514 \pm 21$ & $154 \pm 9$ & $120 \pm 11$ & $588 \pm 22$ \\
Serum albumin & $212 \pm 10$ & $302 \pm 11$ & $215 \pm 8$ & $146 \pm 6$ & $286 \pm 6$ & $20 \pm 3$ \\
\hline
\end{tabular}

Note: Experimental conditions: protein concentration in agar gel, $10 \mathrm{~g} / \mathrm{l}$; protease concentration, $0.3 \mathrm{~g} / \mathrm{l}$; solvent for pepsin: $0.2 \mathrm{M}$ acetate

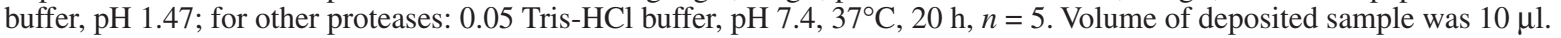

superoxide radical considering the clearly shown involvement of superoxide radical in plasminogen activation by streptokinase [6] failed. For example, ATP and GTP turned out to have comparable efficacies of interactions with the superoxide radical in a model system [7], but only ATP inhibited the activatory function of streptokinase. Longolytin, a fibrinolytic protease from the fungus Arthrobothrys longa, was inhibited in the presence of $0.1 \mathrm{M}$ ATP by $75 \%$ [8]. This ATP concentration is rather high, but further studies of plasminogen-activatory activity of $\beta$ - and $\gamma$-subunits of the nerve growth factor showed that the effective nucleotide concentration was within $0.1 \mathrm{mM}[3,9]$, with the $\beta$-subunit being more sensitive than the $\gamma$-subunit. However, the distribution, mechanism, and the role of this phenomenon are also unclear.

The goal of this work was to reveal specific features of ortho(pyro)phosphate and nucleoside phosphate action on protein cleavage with various proteases and plasminogen-activatory activity of plasminogen activators.

\section{RESULTS AND DISCUSSION}

Proteases cleaved substrate proteins selected for our experiments both in the presence and even in the absence of $P_{\mathrm{i}}, P P_{\mathrm{i}}$, or nucleoside phosphates (control groups). The majority of proteases cleaved gelatin and casein most effectively (Table 1). Plasminogen activators in the absence of the listed effectors initiated lysis of the fibrin gel with the following intensity: streptokinase, $290 \pm 15 \mathrm{~mm}^{2}$; urokinase, $506 \pm 23 \mathrm{~mm}^{2}$; and plasminogen tissue activator, $180 \pm 7 \mathrm{~mm}^{2}$.

Inorganic ortophosphate increased hemoglobin cleavage catalyzed by three serine proteases, particularly, subtilisin by $36-1100 \%^{3}$, gelatin lysis by trypsin and $\alpha$-chymotrypsin by $33-358 \%$, and BSA lysis by subtilisin by $20-92 \%$ (Fig. 1). The effect of $P_{\mathrm{i}}$ on the cleavage of other proteins by these proteinases was not so strong; moreover, there was no effect in the case of casein and fibrinogen. Inorganic orthophosphate enhanced hemoglobin, casein, and BSA degradation

\footnotetext{
${ }^{3}$ Only statistically reliable changes $(P \leq 0.05)$ are given hereinafter.
}

(but not fibrinogen) with papain by $22-76 \%$, whereas, as a rule, it inhibited gelatin lysis. The pepsin-catalyzed proteolysis was enhanced by $45-200 \%$ in the presence of only a low $P_{\mathrm{i}}$ concentration. With an increase in effector concentrations, the pepsin activity was noticeably inhibited up to complete inhibition (for BSA and casein).

The most marked increase in the activity of bacterial metalloprotease in the presence of $P_{\mathrm{i}}$ (with the maximum at $0.03 \mathrm{M}$ ) was observed for hemoglobin degradation (by 44-240\%) and to a lesser extent, for gelatin and BSA. At the maximal $P_{\mathrm{i}}$ concentration, gelatin degradation was inhibited by $44 \%$. Phosphate inhibited the metalloproteinase-catalyzed casein cleavage by $22-47 \%$.

Plasminogen-activatory capacity of all plasminogen activators was increased in the presence of inorganic phosphate by $20-230 \%$, but an especially potent effect was observed for the tissue activator from pig heart (Fig. 2).

Since the addition of phosphates changes in ionic strengths of solutions, we studied the effect of sodium chloride at varied concentrations on gelatin cleavage with proteases [2].

Only trypsin showed the effect analogous to sodium chloride and phosphates in the same range of alterations of the ionic strength. Hence, changes in activities of chymotrypsin, subtilisin, papain, and pepsin in the presence of $P_{\mathrm{i}}$ are not related to changes in the ionic strength of the solution and are specific [2].

Inorganic pyrophosphate. Degradation of a number of proteins by serine proteases increased by 20 $400 \%$ in the presence of $P P_{\mathrm{i}}$ (Fig. 3). For example, lysis of hemoglobin and gelatin with chymotrypsin and subtilisin rose by $28-226 \%$. These proteins were cleaved by $55-250 \%$ more intensely with papain at $P P_{\mathrm{i}}$ concentrations of $\geq 10^{-2} \mathrm{M}$. Degradation of casein and hemoglobin with pepsin and metalloprotease was enhanced by $31-75 \%$ at $P P_{\mathrm{i}}$ concentrations of $10^{-6}$ to $10^{-4} \mathrm{M}$, and of fibrinogen by pepsin, by $44-83 \%$. At the same time, pyrophosphate inhibited by $55 \%$ casein lysis by subtilisin at a concentration of $10^{-1} \mathrm{M}$; BSA lysis with papain at a concentration of $10^{-4}$ to $10^{-1} \mathrm{M}$; and of all proteins with pepsin and bacterial metalloproteinase $\left(10^{-4} \mathrm{M}\right)$. 